Historic, archived document

Do not assume content reflects current scientific knowledge, policies, or practices. 

$\angle 25 \quad \cdots \cdots$

Droad Leaved Batavian, $\begin{array}{r}90 \\ 80\end{array}$

KALE or BORECOLE.

Dwarf Purple Curled, 100 .....

Greén Curled Scotch, 75

KOHL RABI.

Early White Vienna, 150 .....

Purple Vienna,

200

LEEK.

American Flag,

Large Rouen,

$75 \quad \cdots \cdots$

$100 \quad \ldots \ldots$.

Musselburgh,

$100 \ldots \ldots$.

\section{LET TUCE.}

California Cream, $\quad 50 \quad 4500$

Curled Simpson Blk Seed, 504500

". Curled Simpson Blk Seed, 50 45 00

Deacon, $\quad 60 \quad 5000$

$\begin{array}{lll}\text { Early Curleả Silesia, } & 40 & 3500 \\ & 60 & 5000\end{array}$

Imp'd Hanson, Butter,

5000

Paris White Cos(Romaine) 50

Prize Head

salamander

8. 00

4500

5000

4000

Tennisball, Black Seed, $50 \quad 4500$

Tennisball or Boston,

White Seed,

$50 \quad 4500$

Trianon Self-Closing Cos, 60

White Summer Cabbage, $40 \quad 3500$

\section{MELON MUSK.}

Banana,

Bird's Canteloupe

Cassaba (Large Persian),

Delmonico,

Emerald Gem,

Green Citron

Hackensack,

Improved Christiana,

Jenny Lind,

Large Netted Canteloupe 30

Miller's Cream,

Nutmeg,

Shumway, Giant,

Skillman's Netted,

Winter Pine Apple

40

$35 \quad \ldots \ldots$

$35 \quad \ldots \ldots$

. n

$\cdots \cdots$

$\ldots \ldots$

.......

$\ldots \ldots$

$\ldots \ldots$

3000

$\because \ddot{0}$

\section{MELON, WATER.}

Black Spanish,

Boss,

Citron (for preserving),

Cuban Queen,

Dark Icing,

Dixie (the best of all)

Green and Gold,

Ice Cream(Mountain Sweet)35

Jordan's Gray Monarch, 40

Kentucky Wonder,

Kolb's Gem,

Lodi or San Joaquin,

New Christmas,

Phinney's Early (true),

Pride of Georgia,

Ruby Gold.

Southern Rattlesnake,

$35 \quad 3000$

$\begin{array}{lll}30 & 28 & 00\end{array}$

\%

$\begin{array}{lll}25 & 00 \\ : 8 & 00 & 0\end{array}$

$\begin{array}{lll}: 8 & 00 \\ 30 & 00\end{array}$

3500

3000

3500

3000

3000

2200

3000

2800

3500

$\Sigma 000$

\section{MUSHROOM SPAWN.}

English $\}$ Price on application.

MUSTARD.

White or Yellow,

Per lb Per roolbe.

OKRA.

Dwarf Green,

Long Green,

White Velvet,

$40 \quad \cdots \cdots$

$35 \quad \cdots \cdots$

ONIONS.

\section{Barletta}

Extra Early Red,

Giant Red Rocca,

Giant White Tripoli

Queen (Earliest White) 150

Red Glube, Southport, 125

Silver King(Giant White Garganus)

Spanish King (Prizctaker) 150

Wethersfield Large Red, 150

W' hite Globe,

White Portugul,

1 i5

Yellow Danvers, 100 .......

Yellow Globe Danvers $\quad \begin{array}{llll}1 & 10 & \ldots & \ldots .\end{array}$

Yellow Strasburg, 125

\section{ONION SETS.}

Top Onions ? Market Price.

\section{PARSLEY.}

Extra Double Curled,

Fern Leaved,

Moss Curled,

PARSNIP.

Improved Guernsey, $\quad 25 \quad 2000$

Long Smooth, or Hollow

Crowned,

$25 \quad 2000$

Student,

30

PEAS-(Wrinkled Marked thus*) Height

*American Wonder, ${ }^{\text {in }} 109$

Black Eyed Marrowfat:3

Blue Beauty, 208

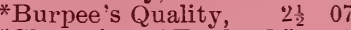

*Champion of England 5

Daniel O'Rourke Imp. $2 \frac{21}{2} 06$

*Everbearing,

First and Best

*Horsford MlrtGarden,

*I_ittle Gem,

*McLean's Advancer, $2 \frac{1}{2} \quad U^{\prime}$

Niles or Small White, 3

*Premium Gem, 1 (8

Pride of the Market, 209

*Stratagem, $1 \frac{1}{2} \quad 07$

Sugar, Dwarf, edible$$
\text { pods, }
$$

$2 \frac{1}{2} \quad 15$

Sugar,Tall, edible pods 5 15

White Marrowfat, 404

*Yorkshire Hero, $_{2} \quad 06$

\section{PE PPERS.}

Golden Dawn, 125

Large Bell or Bull Nose 125

Long Red Cayenne, 150

Red Cherry,

Red Chili, (true)

Ruby King,

Sweet Mountain,

\section{PUMPKIN.}

Connecticut Field or Large Yellow,

Early Sugar,

Large Cheese,

Mammoth Potiron,

-....

$\ldots \ldots$

.....

……

\section{RADISH.}

Black Spanish, long, $\quad 35 \quad$ round,

Chartier improved, long, white tipped

Chinese Rose,

Early White Turnip,

Early Roman Carmine,

French Breakfast,

Long Scarlet Short Top,

Long White Vienna,

Mammo1h White China,

Non Plus Ultra,

Olive Shaped Scarlet,

Scar:et Turnip,

Wlite S1 rasburgh,

Yellow Summer Turnip,

\section{RHUB $4 R B$.}

Linnæus,

Mammoth Monarch, $\quad 100 \quad 75$.

Victoria,

Roots, per: $00, \$ 500$

$\mathrm{SA}$

SIFY aroYSTER PLANT.

White French

Sandwich Island Mammoth $70 \quad \cdots$.

\section{SPINACH.}

Bloomsdale Savoy-leaved $15 \overline{0} \quad 1400$ Long standing, dar'k green, $15 \quad 1300$

New Zealand,

Prickly,

Round thick-leaved,

Viroflay,

1400
1200

1400

\section{SQUASH.}

Boston Marrow, $\quad 35 \quad 3000$

California Field Narrow, $15 \quad 1200$

Early White Bush, $\quad 35 \quad 3000$

Essex Hybrid, $\quad 40 \quad \ldots \ldots$.

Hubbard,

Mammoth Chili,

Marblehead,

Perfect Gem,

Pike's Peak or Sibley,

Jineapple

Summer Crookneck,

Vegetable Marrow,

Winter Crookneck,

4500

-....

n....

(......

3500 。

3

TOMATO.

Acme,

Dwarf Champion,

125

' 150

Favorite, 1 : 10

Natchless,

Perfection, 150

Mikado,

New Stone,

Red Cherry,

Strawberry or Ground

Cherry,

Thorburn's' 250 Thorburn's Long Keeper $175 \quad \ldots \ldots$.

Trophy,

Yellow Plum, $\quad 150 \quad \ldots$. .

TURNIP.

Extra Early Purple Top$$
\text { Milan }
$$

Golden Ball or Orange

$$
\text { Jelly, }
$$

Purple-top Munich,

Red-top Globe,

Red-top Strap Leaf Flat, 30

White Egg,

White Flat Dutch,

White French or Rock,

White Norfolk,

II hite Strap Leaf,

Yellow Aberdeen,

Yellowstone (true),

Rutabiga-Imp. 40

$$
\text { “ Laing's Impr'v'd, } 25
$$


GRASS SEEDS-.

subject to market fluctuations.

Our Selected Grass and Clover Seeds are all of the highest grade of quality, and have been speciall $\frac{y}{y}$ recleaned, and are absolutely free from all noxious weed seeds.

Bermuda Grass (Cynodon Per lb. Per roolbs. dactylon) imported seed 125

Crested Dogstail (Cynosu-

Fescue, Meadow (Festuca

Fescue, Sheep (Festucaovina) 15

Orchard Grass (Dactylis rus cristatus',

Fescue, Slender (Festuca tenuifolia). pratensis),

Fescue, Jard (Festuca duriuscula), Hungarian Grass (Panicum Germanicum

Johnson Grass (Sorghum Halpense)

Kentucky Blue, fancy

clean (poa pratensis),

Millet, common, German, Pearl,

Oat Grass, tall Meadow (Avena elatior), glomerata),

5

40

15

$06 \quad 500$

$10 \quad 800$

$14 \quad 1300$

400

400

2000

1800

Red Top (Agrostis vulgaris), of

Red Top,absolutely clean, 17

(Lolium perenne)

Rye Grass Italian (Lolium

Italicum),

Sweet Vernal, the true perennial sort,

Timothy (Phleum pratense),

Wood Meadow Grass

(Poa nemoralis)

$18 \mathrm{CO}$

700

......

800

10900

50

800
Rye Grass, Perennial
MENLO PARR LAWN GRASS.

A mixture of the varietios

best adapted for the for-

mation of a smooth, vel-

vety $1 \mathrm{a} w \mathrm{n}$, prepared

from the best seeds ob-

tainable, and proved by

reliable tests to be supe-

rior to all other mix-

tures. Per doz. $1 / 2-1 b$ Per lb. Per ionlt:

boxes \$1.75. 2502300

Lawn Grass, fine mixed, $20 \quad 1750$

\section{CLOVER, SEEDS}

Alfalfa,

Crimson Clov

Japan C.over,

d, medium,

d, Manmoth or Pea

Vine,

17

1000

anfoin or Espersette,

White or Dutch,

\section{MISCELLANEOUS.}

Broom Corn, Improved

Evergreen,

Buckwheat,.$\cdots$. " Silver Hulled, 500 Egyptian Corn, white, ......

" brown, ...... 300

Flax Seed, $\quad \ldots \ldots .500$

$\ldots \ldots \circ 50$

$$
\text { grour }
$$

Osage Orange,
Sugar Cane, Early Am-

ber,

Sugar Cane, Early

Orange,

Sunflower, Mammoth

Russian,

Tobacco, Connecticut

Seed Leaf,

Tobacco, Imported

Havana,
600

$40 \quad \ldots \ldots$

500

850

1200

1500

1000

2800

500

400

00

00

00

$700 \quad 600$

$150 \quad \ldots \ldots$

$250 \quad \ldots \ldots$
W.

bers bu.

Bird Seed $a_{2}$

no inistake is.

the cleanest and best

market to-day, and its many known good qualities render it most saleable.

Bird Seed, our celebrated mixture, in cases of $35-1 \mathrm{~b}$. boxes, $\$ 2.00$. $72-1 \mathrm{~b}$ boxes, $\$ 3.75$.

Bone, ground for chickens, per ton, $\$ 35$.

Canary, best Sicily, in original sacks, market price.

Canary, best Sicily, recleaned, market price.

Cuttle Fish Bone,

Hemp,

Per 1b. Per roolbs

ManhattanEgg Food, per case, $252-1$ b pks., $\$ 7.50$.

Maw Seed (Blue Poppy),

Morris' Poultry Cure, per doz. 1-1b. pkgs, $\$ 4 ; 2-1 \mathrm{~b}$. cans, $\$ 7.50$.

Oyster Shells, ground, per ton, $\$ 25$.

Rape, German, $05 \quad \cdots \ddot{7} \dot{75}$

Sunflower, for'Parrots,

Washed Bird Gravel, in bulk, per bbl., price on application.

Washed Bird Gravel, in cases of 2-doz. quart packages, $\$ 1.50$.

\section{$\rightarrow$ Selected Flower Seeds in Bulk.}

\section{FOR FLORISTS' AND DEALERS' USE}

On all orders for packets taken from our retail catalogue, we will allow a discount of 50 per cent. When prices are quoted at 20,25 and 50 cents per ounce, not less than half an ounce, will be sold; for varieties quoted at 75 cents and $\$ 1.00$ per ounce, we will furnish quarter ounces; $\$ 1.50$ and above per ounce, $1 / 8$ of an ounce will be sold at prices quoted; $1 / 4$ pound at pound rates.

Alyssuin, sweet.

Amaranthus, Tricolor (Joseph's Coat),

Antirrhinum, tall mixed,

Aquilegia, double mixed single, mixed,

Aster's, all sorts mixed,

Balloon Vine,

Balsam. Drarf, double mixed,

Calceolaria Grandiflora Hybrida, per 1000 seeds, 50 ets.

Calendula Meteor,

Prince of Orange,

Finest, mixed,

Calliopsis, mixed,

Camelia Japonica

Campanula, mixed

Canary Bird Flower,

Candytuft, climson,

Empress, white,

White Rocket,

Tom Thumb, mixed,

Canna, Crozy's mixed,

Canterbury Bells, single, mixed, double, mixed

Carnation, extra choice, mixed,

Margaret, mixed,

Centaurea candidissima, per 1000 seeds, $40 \mathrm{cts}$,

Centaurea gymnocarpa,

cyanus, mixed

" " Emp. William,

Chrysanthemum, sared from our unrivaled collection of over 500 sorts,

Cineraria हाँचतifora. per 1000 seeds, $\$ 2.00$

Cineraria grandiflora Plenissima, per 100 seeds, 75 cts.

Per oz

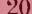

20 25 40 20
Cineraria Maritima (Dusty Miller) 20

Clarkia, fine mixed,

Cobea Scandens.

Coxcomb, choice mixed,

Coleus, splendid mixed, per 1000 seeds, $\$ 1.00$.

Collinsia, mixed,

Convolvulus Major, mixed, Morning Glory, tall,

Convolvulus Minor, mixed, Morning Glory, dwarf,

Cosmos, pink,

$$
\text { “ white, }
$$

Cyclamen Persicum Giganteum, per 100 seeds, $\$ 2.00$

Cypress Vine, scarlet,

" " white,

Dahlia, single, mixed,

"double, mixed,

Daisy, double, mixed,

" Longfellow, pink

Delphinium Formosum,

Dianthus, Chinensis, fl. pl.

$$
\text { " Heddewegii }
$$$$
\text { " Met Laciniatus, fl. pl. }
$$

Digitalis, mixed,

Dolichos, Hyacinth Bean mixed,

Eschscholtza Californica,

$$
\text { Mixed, }
$$

Ferns, greenhouse varities mixed trade pkt., \$i 00

Forget-Me-Not (See Myosotis)

Freesia Refracta Alba,

F'usnia sintro and Amula mixed

per 100 seeds, 50c.

Gaillardia, mixed, 
Pylethrum, aureum (Golden

Salpigossis, mixed,

Balsam Pear,

Musk

Myosotis Alpestris, blue,

" dissitiflora,

palustris (true)

Nemophila, mixed,

Nasturtium, tall, mixed.

Tom Thumb, mixed, Pansy-Azure blue

Bugnot's blotched

Emperor William, (true), 100

Faust

Lord Beaconsfield,

Odier,

Snow Queen,

Trimardeau,

White,

Yellow,

Menlo Park Perfection, 600

Ordinary mixed, 100

Penstemon, mixed,

Perilla nankinensis

Petunia, single, mixed,

double mixed, per $1 \mathrm{c} 00$ seeds $\$ 2.00$.

Phlox Drummondi Grandiflora, mixed,

Poppy,carnation-flowered, mixed Danebrog,

Mikado,

Mephisto,

pæony flowered, mixed

Shirley,

umbrosum

mixed,

Portulaca, single mixed,

" double mixed,

Primula, Chinese, fringed, mixed per 110 seeds, $\$ 200$. Feather)

Ricinus, mixed,

Romneya Coulteri,

Schizanthus, mixed,

Salvia Splendens,

." Patens, per 100 seeds, 50c.

Scabiosa, tall, mixed,

$$
\text { " dwarf, mixed, }
$$

Sensitive Plant,

Silene, mixed (Catchfly)

Smilax

Giant Perfection, mixed,

Wall-flower, leaved, mixed,

Intermediate, crimson,

" Intermediate, crimson,
"“ white,

Sunflower, argophylus, (alifornicus, fl. pl. globosus fistulous. fl. pl. 10 IIammoth Russian,

\begin{tabular}{rr}
\hline Per oz. \\
\\
10 \\
10 \\
100 \\
40 \\
15 \\
1.5 \\
\\
25 \\
30 \\
50 \\
40 \\
15 \\
30 \\
200 \\
\\
350 \\
400 \\
400 \\
300 \\
50 \\
20 \\
10 \\
15 \\
10
\end{tabular}

SWEET PEAS. - W' cultivate more varieties, and control a larger acreage of these than any house in the world.

Per lb.

Alba Magnifica, pure white, $\quad 6(1$

Blanche Ferry, pink and white, 50

Boreatton, crimson purple, very dark 40 Captain of the blues, bright pur-

ple and pale blue

Cardinal, crimson scarlet,

Countess of Radnor, lavender,

crimson 60

Dorothy Tenant, dark mauve,

Duchess of Edinburgh, scarlet flushed with crimson,

Empress of India, rosy pink stand-

$\rightarrow \quad$ ards, white wings

Emily Henderson, pure white,

Fairy Queen, white flushed and feathered with pink

Her Majesty, beantiful rosy pink

Ignea, fiery crimson, scarlet, slightly flushed with purple,

Isa Eckford, creamy white and rosy pink,

Lemon Queen, blush and lemon,
Miss sof pink,

Monareh, bronze, crimson and blue,

Mrs. Elckford, white, delicately sh:ded with primrose,

Mrs. Gladstone delicate soft pink, with rosy wings,

Mrs. Sankey, the purest white; the most perfect form; the largest size, a profuse bloomer, and unsurpassed for florists' use,

Nellie Jaynes, white and light pink,

Orange Prince, orange pink. flushed with scarlet,

Painted Lady, rose crimson and blush white,

Primrose, pale primrose yellow, novel and distinct,

Princess Victoria, cherrs, mauve and pink,

Princess of Wales, white, striped, with lavender and mauve,

Purple Prince, maroon, shaded with purple. wings blue,

Queen of England, white, of large size,

Red and white striped,

dite, , coppery crimson, suf-

fused with rosy pink,

e Queen, bright pink, shaded

Waverly, pale blue and rosy claret,

New Large Flowered, mixed,

Sweet William, double, mixed, single, mixed,

Verbena, mammoth mixed,

" mixed,

Vinca, pure white,

" rose,

" mixed,

Violet, saved from our own unrivaled collection

Virgintan stock, mixed,

Wallfiower, double, mixed single,

Zinnia, Giant Flowered, double, choice mixed,

Our Great Trade Specialty is the already famous "Combination Box A" which contains:

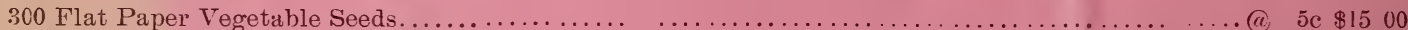

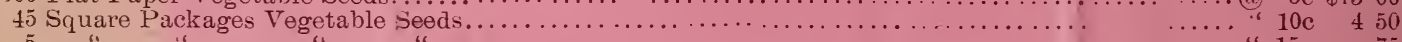

5 " " " "

100 Paper's Assoried Flower Seeds........................................ 5 c 500

WHOLESALE PRICE OF COMBINATION BOX A, $\$ 10.50^{\$}$

The Packages sold by the Sunset Seed and Plant Co., San Francisso, contain NEw SELECTED SEEDS, and have from twenty to fifty per" cent more seed than thos usually sold on commission.

If you do not require the "Combination Box A," you can select Vegetable or Flower Seeds to suit your trade, at the rate of $\$ ! .50$ per hundred packets, if not less than ten of any one variety is ordered at ond time This is exclusive of Peas Berne and Corn, which are put up in $\frac{1}{2}$-poand packages to rotail at ten cənts each, and are wholesaled at $\$ j .00$ per hundred packages.

\section{Miscellaneous Horticultural Requisites.}

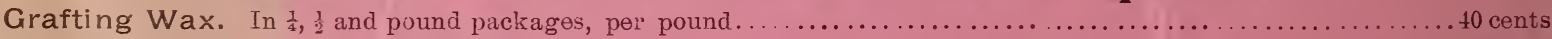
Labels. Plant and tree.-Wooden painted-

Price per thousand..............

$3^{\frac{1}{2}} \quad 4^{1 / 2} \quad 5 \quad 6^{5} \quad 8^{1 / 2}$-inch tree, $\quad 3^{1 / 2}$-inch tree

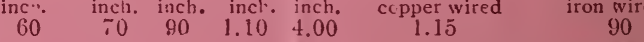

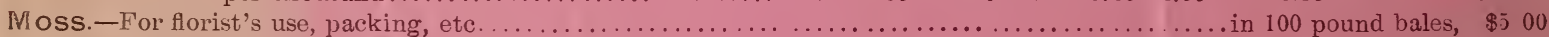
Plant Sticks.-Painted Green-

Pruner. "The LeviN." The strongest hand shear extant. It is made of the finest steel, and though only $i$

6 feet.

ounces in weight, it is so strong that it can cut through half an inch of dry oak.............. 90ets. each

Raffia. Ior tying plants: superior to twine.............................. per $1 \mathrm{~b} ., 20$ cents; per $100 \mathrm{ibs} \ldots \ldots \ldots \$ 1600$

Whale Oil Soap. Excellent wash for trees and plants to prevent insects from lodging in the bark, and for smearing on the trunks of trees to prevent worms from crawling up. Por i-lb. tins, 15 cts.; in bulk, in quantities of 10 lbs. or over

8 cents per pound.

\section{Sunset Seed and Plant Co., San Francisco, Cal.}

